\title{
Vingt ans de recherches à Saint-Véran, Hautes Alpes: état des connaissances de l'activité de production de cuivre à l'âge du Bronze ancien
}

\author{
Veinte años de investigaciones en Saint-Véran, Hautes-Alpes: estado de la cuestión sobre \\ la producción del cobre durante la Edad del Bronce Antiguo
}

\author{
David Bourgarit (*) \\ Pierre Rostan (**) \\ Laurent Carozza $(* * *)$ \\ Benoît Mille (*) \\ Gilberto Artioli (****)
}

\section{RÉSUMÉ}

A l'âge du Bronze ancien en Europe occidentale, l'augmentation drastique de la production de cuivre est très peu documentée au niveau des activités minières et métallurgiques de transformation. Dans ce contexte, le gisement minier et métallurgique de Saint-Véran, Hautes-Alpes constitue un témoin privilégié de la production de masse à la fin du $3^{\mathrm{e}}$ millénaire, puisqu'aux travaux miniers rendant compte d'une exploitation à grande échelle, avec une production de près de sept tonnes de cuivre par an, plusieurs sites de métallurgie extractive ont pu être associés. Depuis près de vingt ans, plusieurs équipes dont celle rassemblant les présents auteurs ont mené ou mènent des recherches pour tenter de documenter le site et son activité. L'objectif de cet article est de présenter l'état de nos connaissances du gisement au jour d'aujourd'hui. Les résultats principaux sont la mise en évidence et la quantification de l'activité de production de masse. De plus, une première description de l'organisation de la production a pu être donnée, où sont mis en lumière les phasages entre activité minière et métallurgiques, ainsi que les techniques métallurgiques innovantes mises en place. Le gisement révèle un éclatement très marqué de l'activité métallurgique dans l'espace qui ne laisse pas d'interroger. De même, la question de la destination de la production reste entièrement posée. Par ailleurs, les moteurs d'une telle implantation

(*) C2RMF UMR 171 du CNRS, Palais du Louvre - Porte des Lions, 14 quai François Mitterrand, 75001 Paris, France. Correos electrónicos: david.bourgarit@culture.gouv.fr and benoit.mille@culture.gouv.fr

(**) Bureau d'Études Géologiques TETHYS, 05380 Châteauroux-les-Alpes, France. Correo electrónico: roc2@wanadoo.fr> en territoire de haute montagne ont commencé d'être identifiés. Ainsi sont évoqués, pour les seuls déterminismes naturels, aussi bien la minéralisation particulière du gisement que l'évolution du climat.

\section{RESUMEN}

Durante la Edad del Bronce en Europa occidental, el aumento drástico de la producción de cobre apenas está documentado en las actividades mineras y metalúrgicas de transformación. En este contexto, el yacimiento minero y metalúrgico de Saint-Véran, Hautes-Alpes constituye un testimonio privilegiado de la producción en masa a fines del III milenio, ya que las obras mineras dan cuenta de una explotación a gran escala, con una producción de casi 7 t de cobre al año. Numerosos sitios de metalurgia extractiva puede haber estado asociados con ella. Desde hace 20 años, diversos equipos, como en el que participan los autores, han emprendido o emprenden investigaciones para intentar documentar el sitio y su actividad. El objetivo de este artículo es presentar el estado actual de la cuestión sobre el yacimiento. Los resultados principales son la puesta en evidencia de la actividad de producción en masa y su cuantificación. Además se ha descrito por primera vez la organización de la producción,

(***) UMR 5602 Géographie de l'Environnement (CNRS/UTM), Maison de la Recherche, allées Antonio Machado, 31000 Toulouse, France. Correo electrónico:

laurent.carozza@univ-tlse2.fr

(****) Dipartimento di Geoscienze, Università di Padova,Via Giotto 1, I-35137 Padova, Italy. Correo electrónico: gilberto.artioli@unipd.it

Recibido: 11-III-2010; aceptado: 20-V-2010. 
poniendo en evidencia la sucesión de fases entre la actividad minera y metalúrgica, así como las técnicas metalúrgicas innovadoras puestas en práctica. El yacimiento revela un estallido muy marcado de la actividad metalúrgica en el espacio que no deja de plantear interrogantes. Igualmente, la cuestión del destino de la producción sigue abierta por completo. Por otro lado, los motores para una implantación semejante en un territorio de alta montaña han comenzado a identificarse. Así se invocan determinismos naturales ya se trate de la mineralización particular de un yacimiento como de la evolución del clima.

Mots clé: Âge du Bronze ancien; Alpes occidentales; Métallurgie; Cuivre.

Palabras clave: Edad del Bronce Antiguo; Alpes occidentales; Metalurgia; Cobre.

\section{INTRODUCTION}

Le gisement de Saint-Véran, situé dans la partie méridionale des Alpes occidentales près de la frontière avec l'Italie (Fig. 1), a vu se développer dès le début de l'âge du Bronze ancien une exploitation de cuivre à grande échelle. L'étude de la mine de la "tranchée des anciens" rend compte d'une augmentation très importante de la pro-

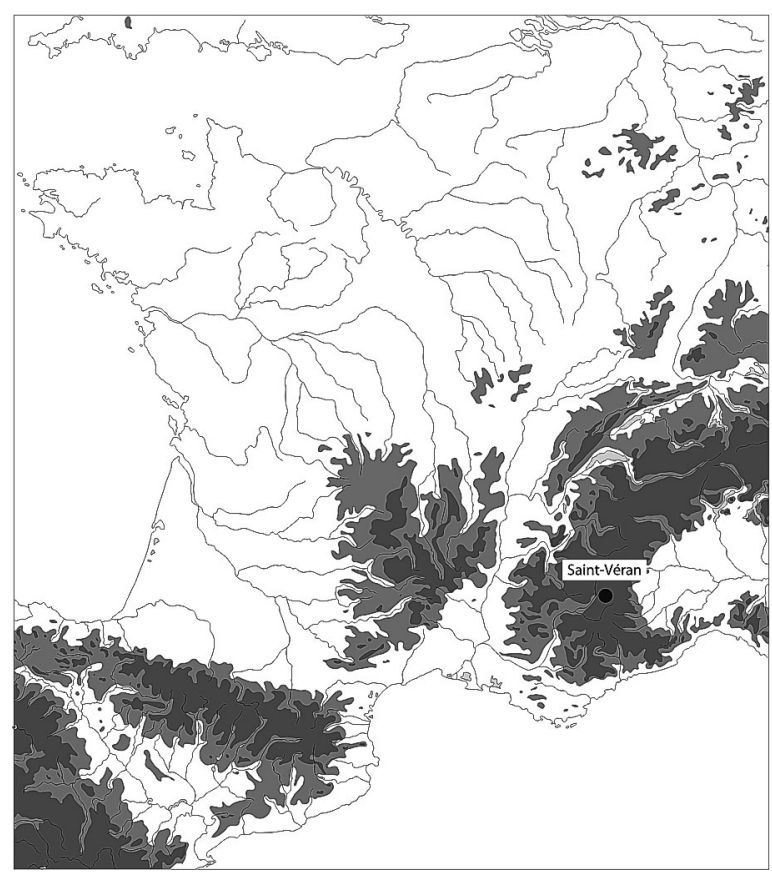

Fig. 1 Carte de localisation du gisement de Saint-Véran, Hautes-Alpes (haut Queyras). duction de cuivre par rapport aux périodes précédentes du Chalcolithique, estimée à environ sept tonnes par an (Rostan et al. 2002) (1). L'étude archéologique et la caractérisation physico chimique des témoins de traitement métallurgique contemporains de l'exploitation minière (Barge 1997; Bourgarit et al. 2008; Ploquin et al. 1997) (2) ont, quant à elles, mis en évidence un niveau technologique des pratiques métallurgiques remarquablement élevé.

Si de tels bouleversements dans les modes de production du cuivre au début de l'âge du Bronze sont connus et relativement bien documentés pour le Proche-Orient (Hauptmann 2000; Rothenberg 1990), peu de données existent pour l'Europe et notamment l'Europe occidentale. Dans ce contexte, le gisement minier et métallurgique de Saint-Véran constitue un ensemble clé, qui a rassemblé depuis près de vingt ans de nombreux chercheurs venus d'horizons variés. Le présent article se propose de faire un point des connaissances acquises depuis le début des travaux de recherche.

\section{PRESENTATION GENERALE DU GISEMENT}

Depuis 1994, les différentes prospections de surface, sondages et fouilles ont mis en évidence quatre sites liés à la production de cuivre au début de l'âge du Bronze ancien (Fig. 2).

La tranchée des anciens, une exploitation de minerai de cuivre hyposulfuré, la bornite, située dans la haute vallée de l'Aigue Agnelle dans la partie orientale du massif du Queyras a été fouillée en 1995-1996 (Ancel 1997; Rostan et al. 2002). Une datation réalisée sur un élément de poutraison de la colonne orientale permet de dater ces travaux de l'âge du Bronze ancien (AA.27533 $3635 \pm 80$ BP soit 2300-1750 BC).

Le site minier comporte également un abri, l'abri de Pinilière (Rostan and Malaterre 1994b), situé sous un grand bloc erratique de gabbro avec

(1) Le caractère massif du gisement, la simplicité du minerai (bornite pure) et la simplicité du gite ont permis de donner un ordre de grandeur tant de la quantité de métal produite que du temps nécessaire à l'extraction du minerai.

(2) Voir aussi Burger, E. 2008: "Métallurgie extractive protohistorique du cuivre: Etude thermodynamique et cinétique des réactions chimiques de transformation de minerais de cuivre sulfurés en métal et caractérisation des procédés". Unpublished $\mathrm{PhD}$ Dissertation, University Paris VI. 


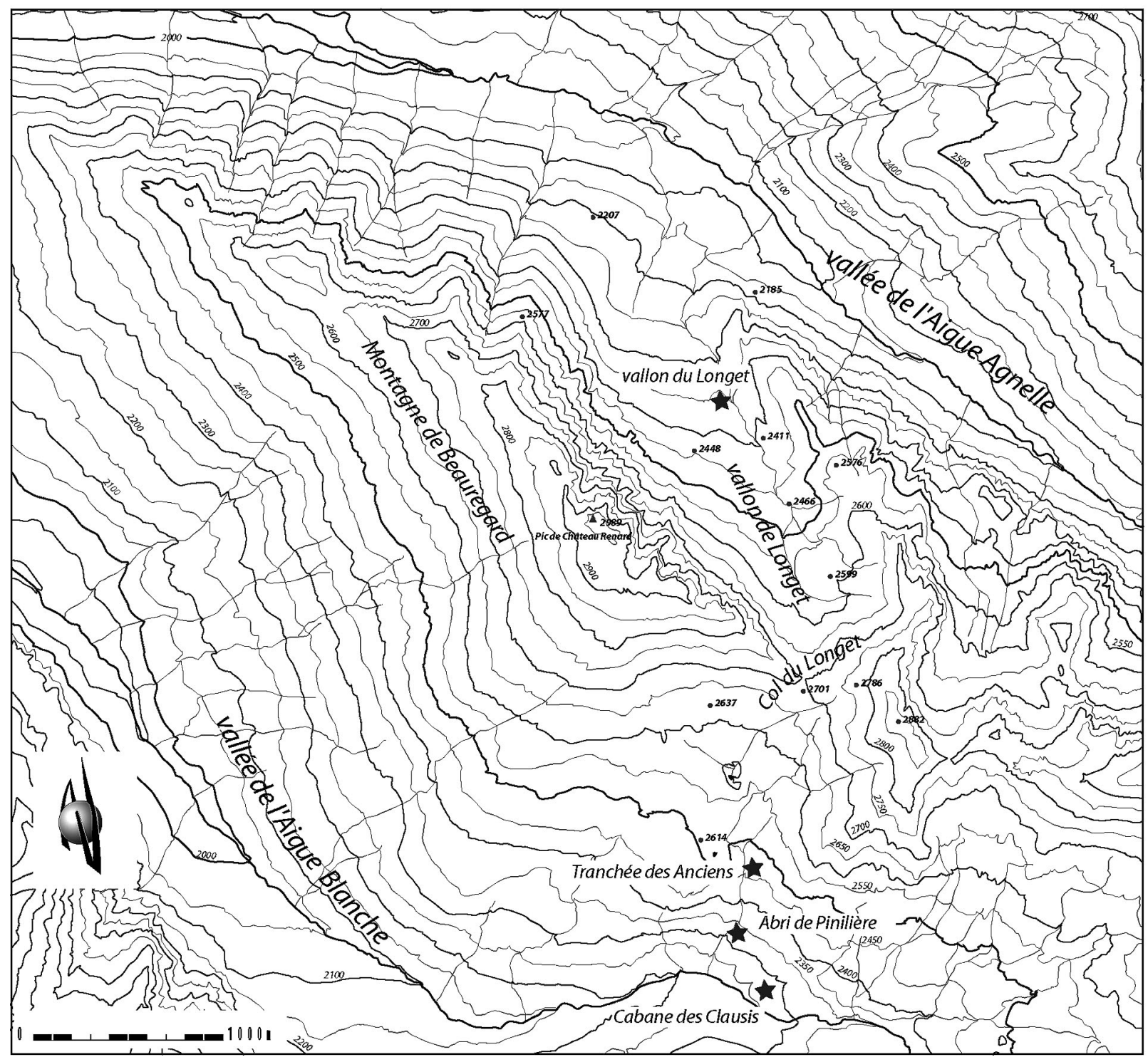

Fig. 2. Carte des principaux sites liés à l'exploitation des ressources cuprifères durant l'âge du Bronze dans la zone de Saint-Véran et de Molines-en-Queyras

trois phases d'occupation dont la plus ancienne est liée à l'exploitation minière et plusieurs aires de traitement du minerai.

Située environ $350 \mathrm{~m}$ en aval de la zone minière, la plate-forme des Clausis a livré un ensemble de témoins archéologiques relatifs aux activités métallurgiques de traitement de minerai (Barge 1997), nous reviendrons plus en détail sur ce mobilier par la suite. Dans les fondations du bâtiment sont apparus les premiers vestiges archéologiques, des résidus carbonisés (charbons de bois et cendres) associés à des scories for- maient un amas polyphasé. Ces dépôts sont caractérisés par l'alternance d'horizons limonosableux et caillouteux et de niveaux riches en produits de combustion. L'implantation de la tranchée de fondation de la cabane des Clausis ne permet malheureusement pas, dans cette zone, d'établir le lien stratigraphique de ces vidanges avec une structure de combustion. L'extension de la fouille a mis en évidence, dans toute sa complexité, l'établissement métallurgique à proprement parler. Les horizons archéologiques se présentent sous la forme d'un palimpseste de len- 
tilles imbriquées. Les fouilleurs ont ainsi pu décrire différents horizons incluant de manière plus ou moins dense des produits de combustion et des résidus de traitement métallurgique du minerai. En l'absence de structures de combustion en relation directe avec cette zone, la distribution des artefacts et des produits semble plutôt illustrer les activités de concassage autour de tables de broyage. Les datations au C14 sur charbons de bois indiquent une date comprise entre 2555 et 2029 cal. (3760 65 BP).

Enfin, un second site de traitement métallurgique, le site du Vallon du Longet, à Molines-enQueyras, a été découvert lors de prospections antérieures à 1995, réalisées dans le cadre des travaux de recherche engagés sur le site minier des Clausis (Rostan et al. 2002). Un des intérêts du site du vallon du Longet réside dans son éloignement relatif d'avec le site d'extraction du minerai, qui est situé plus au nord du site minier au-delà du col du Longet (Fig. 2). Deux sondages menés en 2007 et 2008 (Carozza et al. 2010a) ont permis de montrer que les premiers horizons archéologiques reposent sur le substratum, formé ici par une moraine argilo-graveleuse jaunâtre. La relative horizontalité des dépôts sédimentaires qui forment les niveaux archéologiques sous-tend que le site est implanté sur un replat adossé au versant. Plusieurs artefacts signent clairement une activité de métallurgie extractive, et bien que le site soit très mal conservé et n'ait livré qu'un mobilier peu abondant, ces quelques éléments montrent sans conteste que l'on a affaire à un site similaire à celui de la cabane des Clausis. Les datations attestent d'une occupation à la fin du $3^{\text {ème }}$ millénaire avant notre ère (2280-2030 BC).

La compilation récente (Carozza and Mille 2007; Mille and Carozza 2009) de toutes les dates C14 obtenues sur la Cabane des Clausis et la tranchée des anciens montre que les fonctionnements ont été très probablement contemporains (Fig. 3). Ainsi, la plage de temps couverte par les datations obtenues dans différents horizons du site des Clausis s'étend entre la seconde moitié du $26^{\text {ème }}$ siècle avant notre ère et le 18ème siècle avant notre ère. La durée statistique représentée englobe l'extrême fin du Néolithique et l'âge du Bronze ancien. Elle recouvre l'ensemble du Campaniforme. Si l'on cumule les dates et leurs plages de probabilité par demi-siècle, la période comprise le $20^{\text {ème }}$ et le 24 ème siècle avant notre ère est prépondérante. La datation obtenue récem-

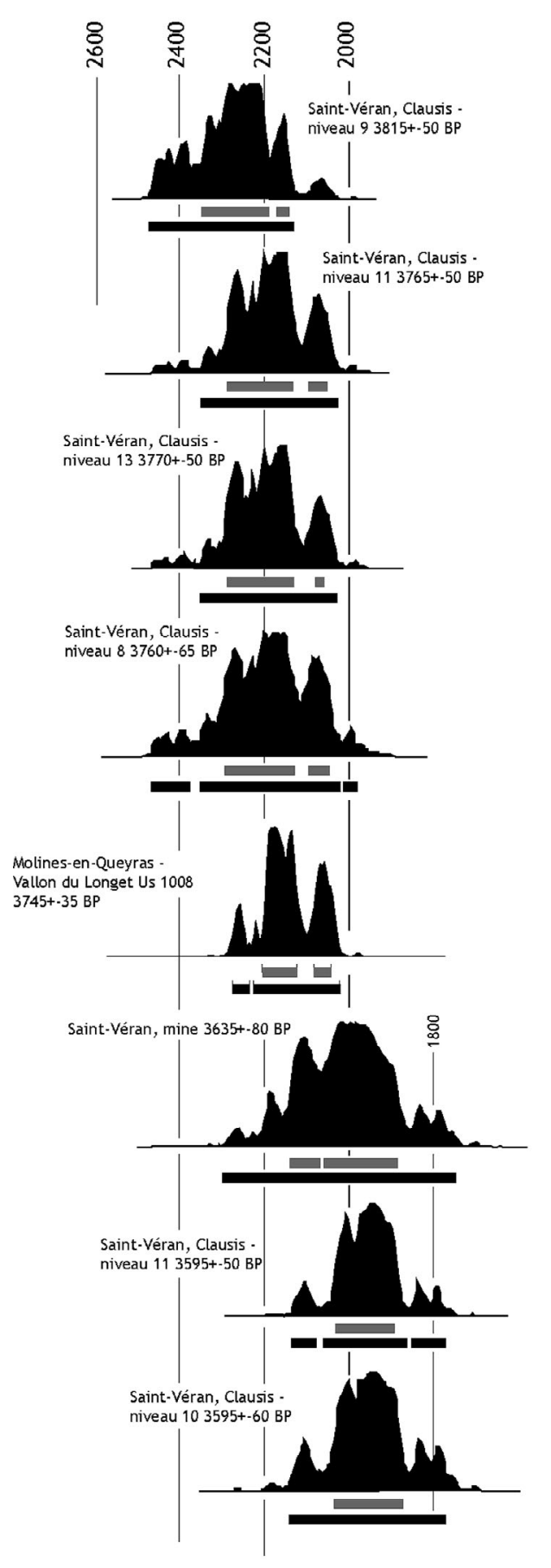

Fig. 3. Compilation des principales datations obtenues par C14 sur la mine "La tranchée des anciens", sur le site de la Cabane des Clausis, et sur le site du Vallon du Longet d'après (Carozza and Mille 2007; Carozza, et al. 2008). 
ment sur le site du vallon du Longet vient conforter ces observations (Us 1008 - Poz-22369 $3745 \pm 35 \mathrm{BP}$ ) et fournit une fourchette chronologique comprise entre 2280-2030 BC, correspondant au début du Bronze ancien.

D'un point de vue culturel, les mobiliers ne permettent ni d'affiner cette perception, ni d'identifier de particularités culturelles. L'importance de la sphère italique a cependant été relevée par Pierre Rostan et Maurizio Rossi, nous y reviendrons.

\section{MINE ET MINERAIS}

\section{Le gisement}

Il s'agit d'un gisement sédimentaire exhalatif mis en place sur le fond océanique de la Téthys, composé ici de gabbros, chloritoschistes et serpentinites (3) qui est aujourd'hui redressé avec une allure pseudofilonienne et dont l'allure se trouve influencée par la tectonique alpine. Le corps minéralisé consiste dans une couche plus ou moins épaisse de sulfures massifs avec $\mathrm{Cu}$, $\mathrm{Zn}, \mathrm{Fe}$, etc. avec traces de $\mathrm{Pt}, \mathrm{Te}, \mathrm{Au}, \mathrm{Ag}, \mathrm{Co}$, $\mathrm{Sn}$.

Le gîte comporte deux branches aux affleurements exploités à la préhistoire et les travaux miniers situés en haute altitude ( $2.400 \mathrm{~m}$ et plus) s'y sont développés sur une profondeur importante, de l'ordre de $80 \mathrm{~m}$ (Rostan et al. 2002) pour l'exploitation de la bornite $\left(\mathrm{Cu}_{5} \mathrm{FeS}_{4}\right)$ pour être poursuivis plus en profondeur au $20^{\text {ème }}$ siècle. L'examen des travaux miniers a montré que la bornite avait été récupérée partout avec le plus grand soin et les vides laissés par l'exploitation préhistorique comme les remblais intérieurs ou les haldes sont particulièrement pauvres en minerai.

Les travaux miniers préhistoriques, accessibles en profondeur par suite de la poursuite de l'exploitation du gîte au 20 ème siècle, sont encore demeurés inaccessibles près de la surface à la partie supérieure du gisement.

(3) Ayoub 1994: "Un exemple de minéralisation associée aux ophiolites mésozoïques des Alpes Cottiennes: le gîte de Saint-Véran (Hautes-Alpes, France). Etude structurale, pétrographique et métallogénique". Unpublished $\mathrm{PhD}$ thesis. University of Grenoble, France.

\section{Les minerais}

La bornite $\left(\mathrm{Cu}_{5} \mathrm{FeS}_{4}\right)$ se présente principalement dans le gisement sous forme d'une bande subverticale de minerai massif d'épaisseur au moins décimétrique entre des chloritoschistes tendres du socle océanique (et parfois des serpentinites) et des quartzites massives (anciennes radiolarites métamorphisées); le minerai est nettement distinct d'avec les formations encaissantes dont il est parfaitement séparé et atteint parfois jusqu'à plus de 20 ou $30 \mathrm{~cm}$. Cet aspect confère au gisement un caractère tout à fait exceptionnel car les sites comportant ainsi de tels sulfures riches et massifs accessibles au mineur préhistoriques sont tout à fait exceptionnels; le gîte de Saint-Véran, sur un plan purement minier, ne peut être mis en concurrence avec les autres gîtes sulfurés régionaux ou français, en particulier avec les filons de quartz à chalcopyrite du massif alpin où le minerai, plus pauvre, est dispersé dans une gangue très tenace.

La richesse en cuivre métallique de ce minerai (de l'ordre de $45 \%$ ) résulte du caractère hyposulfuré du gisement et la chalcopyrite n'y présente ainsi qu'un caractère très accessoire. Le minerai est souvent associé à la chalcocite et à des oxydes de fer; riche en blende, il comporte de nombreuses inclusions de tellures, notamment d'or. Des analyses élémentaires et de rapports isotopiques $\mathrm{du}$ plomb ont été réalisées très récemment sur plusieurs minéralisations de cuivre associées au gisement ancien. Une première présentation des résultats des analyses isotopiques apparaît dans (Artioli et al. 2009).

Un autre acquis des recherches récentes est d'avoir mis en lumière la présence récurrente de cuivre natif à Saint-Véran. D'une part, lors de la réalisation des travaux modernes en 1940 du cuivre natif a été rencontré très en profondeur dans le gisement vers 1940 au niveau du travers-banc n. ${ }^{\circ} 4$ avec de la réalisation des travaux modernes sous forme d'une lentille de plusieurs centaines de kilos, indépendante de la minéralisation sulfurée. Toutefois, un examen attentif des fonds d'archives montre que celui-ci avait été rencontré en surface lors du percement du travers-banc $n .^{\circ} 0$ en partie amont du site minier, et des morceaux importants de cuivre métallique sont également cités dans la littérature du $19^{\text {ème }}$ siècle.

De plus, des fragments de cuivre natif de quelques centimètres ont été rencontrés dans les hal- 
des préhistoriques lors des tentatives mécanisées de dégagement du travers-banc $n^{\circ} 2$ bis en 1993. Enfin, des travaux de terrassement réalisés ces dernières années sur le site minier ont mis au jour un morceau de cuivre natif de $180 \mathrm{~g}$ dans les haldes préhistoriques de la "Tranchée des Anciens".

Il semblerait donc que le gîte ait comporté des quantités notables de cuivre natif dès la surface, issus de phénomènes de cémentation consécutifs à l'altération météorique et superficielle, et que la présence de ce cuivre natif soit demeurée inaperçue par suite de l'importance des travaux préhistoriques et de l'impossibilité actuelle de pénétrer les zones de travaux les plus proches de la surface du gîte.

Cet aspect apparaît donc plus nuancé que ce que nous avions formulé par le passé, et SaintVéran pourrait rejoindre le site minier de Roua (Rostan and Mari 2005a) dans la liste des rares exploitations protohistoriques françaises de cuivre natif.

Les quantités importantes de cuivre natif retrouvées en contexte secondaire à Saint-Véran, et en particulier dans les anciennes haldes minières suggèrent que ce type de minerai a également été exploité anciennement, sans qu'il soit pour l'heure possible de phaser cette exploitation par rapport à celle de la bornite, à l'âge du Bronze ancien. Il est en tous les cas pour le moins tentant de considérer cette source "facile" de cuivre, ne requérant que peu sinon aucun traitement pyrométallurgique, comme ayant servi de déclencheur à l'exploitation minière intensive de bornite et la production de cuivre de masse qui en a résulté.

Il est important de signaler que les produits secondaires habituels issus de l'altération superficielle des sulfures (oxydes et carbonates) semblent faire totalement défaut dans le gisement.

\section{MOBILIER MINIER ET METALLURGIQUE}

\section{Mobilier et outillage}

Parmi les deux sites de traitement métallurgique fouillés, le site de la cabane des Clausis a fourni l'essentiel du mobilier lié à la métallurgie (Barge 1997).

Percuteurs, pierres à cupule, mortiers, constituent l'essentiel des outils. Ces éléments se rapportent aux activités d'enrichissement du minerai, de broyage des scories et des produits de fusion. Les tuyères en céramique, très nombreuses et fragmentées, sont de morphologie spécifique (Fig. 4). Il s'agit de pièces de forme conique en céramique fine qui se sont révélées dans l'ensemble très homogènes sur le plan géométrique, même si des nuances morphologiques peuvent apparaître. Courtes, au profil galbé, avec une longueur modérée (50 à 60 mm estimés) pouvant atteindre $88 \mathrm{~mm}$, elles présentent une extrémité distale évasée et épaissie. Le canal central présente une extrémité distale de diamètre interne variant de 10 à $20 \mathrm{~mm}$ en général (jusqu'à $33 \mathrm{~mm}$ pour un individu) et une extrémité proximale, coté foyer, de faible section n'excédant pas 3 à 4 $\mathrm{mm}$. Les parois sont peu épaisses, de l'ordre de 4 à $10 \mathrm{~mm}$ au plus. Les très nombreux fragments de tuyères rencontrés en fouille ne montrent quasiment jamais de traces de scorification, attestant d'une position haute de l'apport d'air par rapport au foyer. Des pièces analogues ont été mises au jour dans l'abri de Pinilière qui jouxte la mine protohistorique (Gattiglia and Rossi 1995; Rostan and Malaterre 1994b; Rostan et al. 2002), ainsi qu'en différents points du territoire valléen (Rostan, inédit). Le site du vallon du Longet a livré un fragment de tuyère tout à fait comparable.

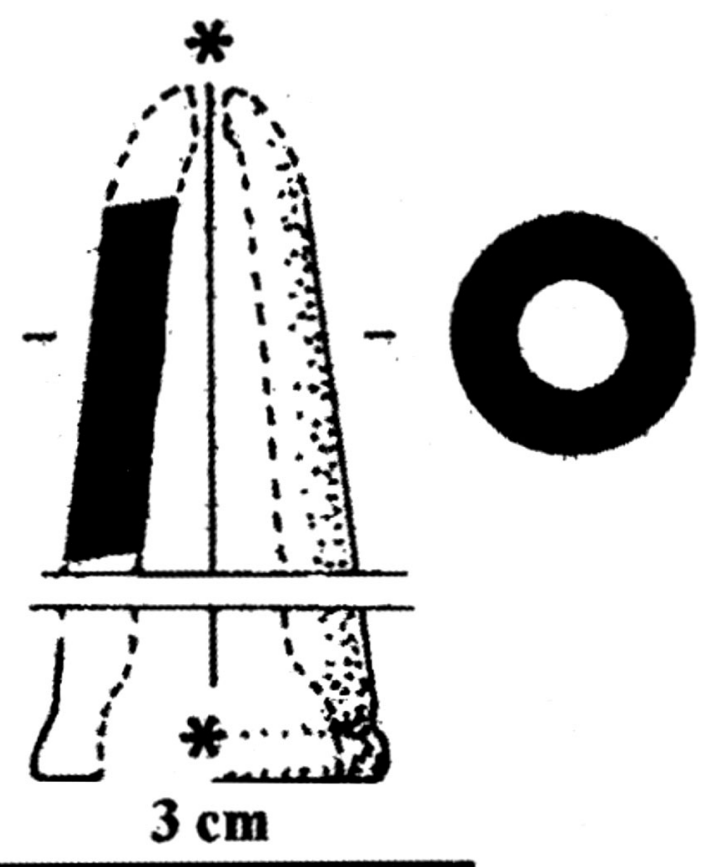

Fig. 4. Exemple de tuyère trouvée à Saint-Véran dans un contexte de l'âge du Bronze ancien (d'après (Rostan et al. 2002)). 
Par ailleurs, la Cabane des Clausis a livré un étonnant creuset brisé en phlogopite massive (Barge 1997). Là, des structures de broyage y ont été identifiées (Ancel 1998). De plus, les premiers travaux de fouille entrepris dès la découverte du site (Gattiglia and Rossi 1995) ont mis en évidence la présence, en partie amont, de deux structures de combustion situées de part et d'autres d'un grande dalle de calcschistes; malheureusement, la structure principale n'a pas été fouillée et se trouve à présent sous la semelle de fondation en béton d'une cabane d'alpage.

$\mathrm{Au}$ Vallon du Longet, le sondage-diagnostic réalisé en septembre 2007 a mis au jour un fragment de schiste (quartzophyllade) de $5 \times 2 \times 4 \mathrm{~cm}$ présentant une surface vitrifiée (Fig. 5). L'étude pétrographique et chimique a montré qu'une mince couche d'un matériau analogue aux scories était soudée à la roche, avec en particulier la présence de fayalite riche en magnésium indiquant une température atteinte comprise entre 1.200 et $1.300^{\circ} \mathrm{C}$. Ceci montre sans ambigüité que ce fragment provient d'une paroi de four métallurgique. Cet indice nous renseigne de manière indirecte sur l'existence de structures bâties dévolues aux activités pyro-métallurgiques. Bien que cette hypothèse ait été présentée par Pierre Rostan dès la découverte du site de la cabane des Clausis à Saint-Véran, les fouilles successives n'avaient pas permis d'en démontrer l'existence, jusqu'à cette découverte récente.

\section{Scories}

Au sein du corpus mis au jour, les morphologies des scories sont très homogènes, la plus grande concentration se trouvant, pour l'heure, à la Cabane des Clausis (une centaine de kilos environ, contre 14 fragments de scories au Vallon du Longet représentant moins de $250 \mathrm{~g}$ ). Ainsi, plus de $98 \%$ des scories de La Cabane des Clausis (Ploquin et al. 1997) et toutes les scories du vallon du Longet sont les témoins les plus anciens d'un type très particulier de déchets métallurgiques, les Plattenschlacke (Fig. 6). Ces scories sont plates, d'épaisseur variant de 8 à $20 \mathrm{~mm}$, fragmentaires (de l'ordre du $\mathrm{cm}$ ), et massives et très peu poreuses, et de grande densité $(\sim 3,5$ g. $\mathrm{cm}^{-3}$ ). Elles témoignent d'une fusion complète; ainsi on ne rencontre que très rarement des restes de quartz non fondu. La surface supérieure est très lisse tout en présentant quasi systématiquement des plis en "peau de lait", tandis que des gouttes de cuivre oxydé apparaissent sur certains échantillons, de même que des impressions de charbon en négatif. La surface inférieure est nettement plus rugueuse et comporte de nombreuses
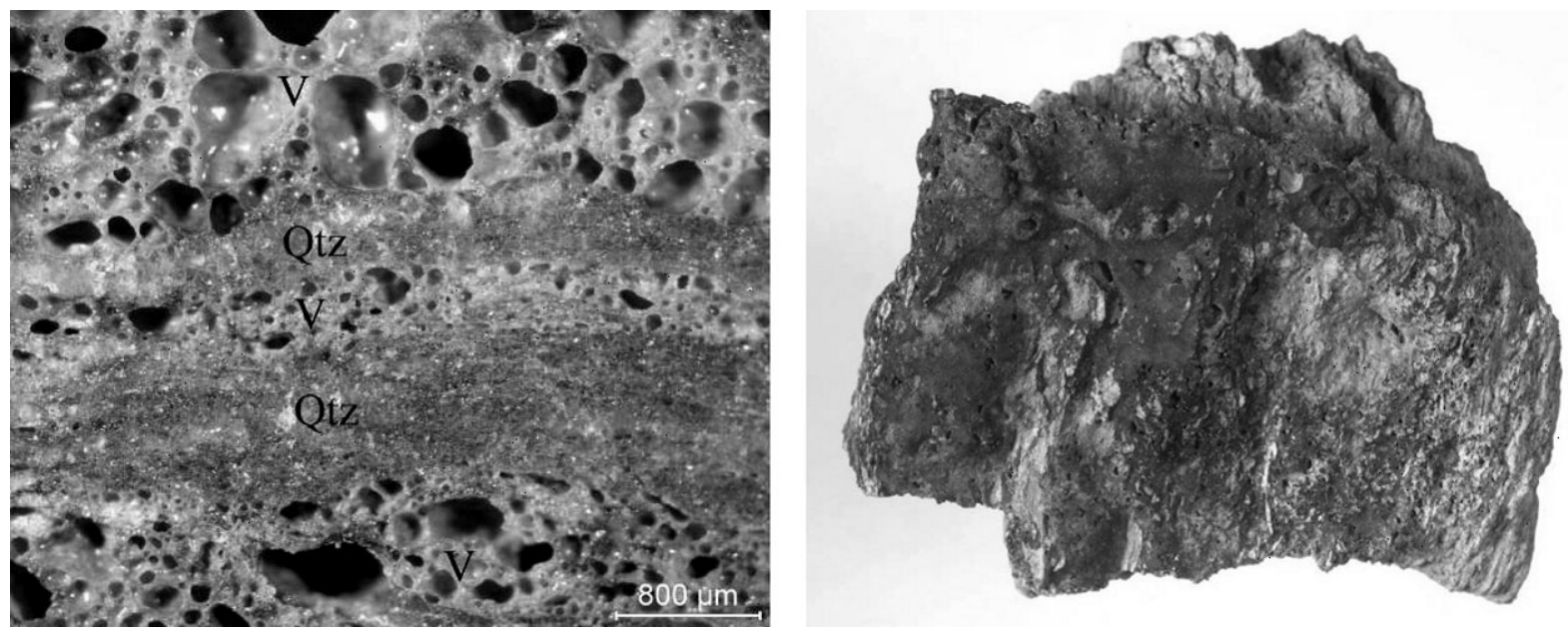

Fig. 5. Vues de dessus (for the editor: haut ou gauche, largeur du fragment environ $5 \mathrm{~cm}$ ) et en coupe après section (for the editor: bas ou droite) du fragment de cloritoschiste mis au jour sur le site du Vallon du Longet présentant une surface vitrifiée (en noir). L'étude de la microstructure de la zone vitrifiée montre qu'il s'agit très certainement d'une paroi de structure de combustion liée à la transformation pyrométallurgique du minerai de cuivre (photos Yvan Coquinot, C2RMF). 


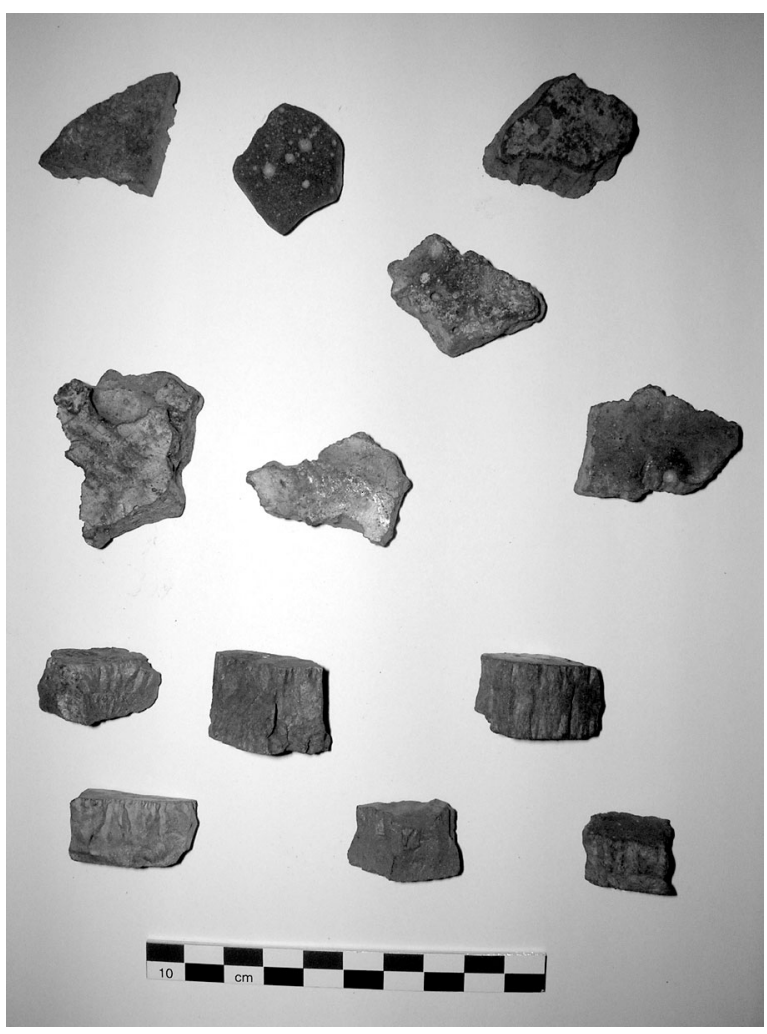

Fig. 6. Fragments de scories retrouvés sur différents sites métallurgiques de Saint-Véran de l'âge du Bronze ancien montrant la morphologie caractéristique des Plattenschlacke.

porosités. Le diamètre des scories à la Cabane des Clausis a pu être estimé à partir de la courbure de nombreux fragments de bords à 150-200 $\mathrm{mm}$. On signalera qu'au Vallon du Longet, une des scories présente un bord droit laissant plutôt envisager une morphologie quadrangulaire. Quelques rares scories à La Cabane des Clausis présentent des structures beaucoup plus poreuses.

Ce type de scories n'étaient jusqu'alors attestées que dans des contextes alpins beaucoup plus tardifs, à l'âge du Bronze moyen et final eg (Herdits 1993; Herdits 2003). En comparaison, les scories néolithiques (début du 3 ème millénaire avant JC) de Roque-Fenestre et La Capitelle du Broum, Hérault (Bourgarit and Mille 1997; 2005; Bourgarit et al. 2003) sont des matériaux relativement peu denses (autour de $2,5 \mathrm{~g} . \mathrm{cm}^{-3}$ ), partiellement fondus et donc très hétérogènes, révélant des procédés métallurgiques immatures typiques des métallurgies néolithiques et chalcolithiques (Bourgarit 2007). Il est de plus intéressant de sig- naler que de telles scories ont également été retrouvées sur le site de Al Claus, Tarn et Garonne (Carozza et al. 1997), dont l'occupation est synchrone de l'activité métallurgique protohistorique de Saint-Véran $(3855 \pm 45 \mathrm{BP}$, soit 2448-2175 av. J.-C.).

Un autre aspect macroscopique des plus intéressants des scories retrouvés aux Clausis, pourtant non remarqué lors des études anciennes, est la morphologie colonnaire de la partie supérieure de la surface de fracture des fragments de scories (Fig. 7). Quelle que soit l'épaisseur de la scorie, une morphologie prismatique apparaît systématiquement sur les 4-6 premiers millimètres sous la surface supérieure. Sur les fragments correspondant aux bords de la scorie, cette structure particulière émane des deux surfaces, supérieure et inférieure. Quelques rares exemples ne présentent pas cette caractéristique morphologique: ce sont les fragments très fins. De tels faciès n'ont quasiment pas été observés au Vallon du Longet, bien qu'on ne peut pas exclure que la corrosion ait érodé les scories.

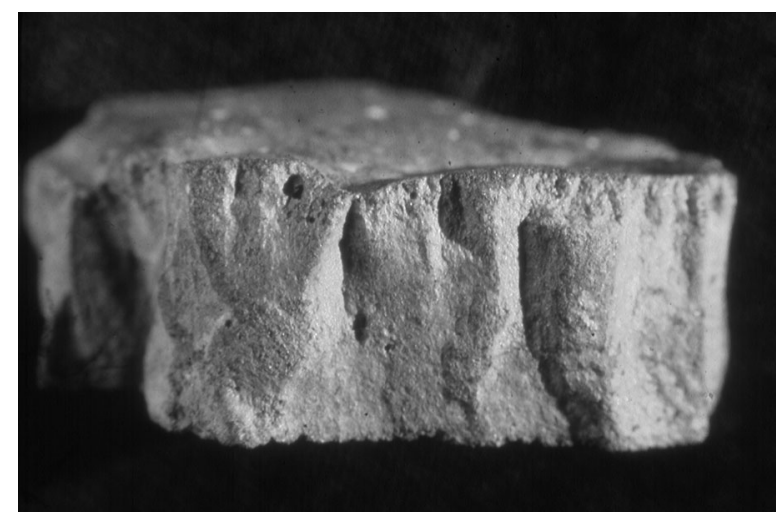

Fig. 7. Vue sur la tranche d'un fragment de scorie de Saint-Véran montrant, sur la surface de fracture, la couche de morphologie prismatique qui occupe les 1-2 premiers millimètres sous la surface supérieure (en haut). L'épaisseur totale de la scorie est de l'ordre de $10 \mathrm{~mm}$.

Le détail des études minéralogiques et chimiques est présenté ailleurs (Bourgarit, et al. 2008; Ploquin, et al. 1997), nous nous contenterons ici d'en rapporter les principaux résultats. Toutes les scories étudiées montrent des compositions très similaires situées autour du domaine fayalitique (Fig. 8), avec des taux de cuivre résiduel variant de 1 à $11 \%$ en masse, et une teneur globale en 


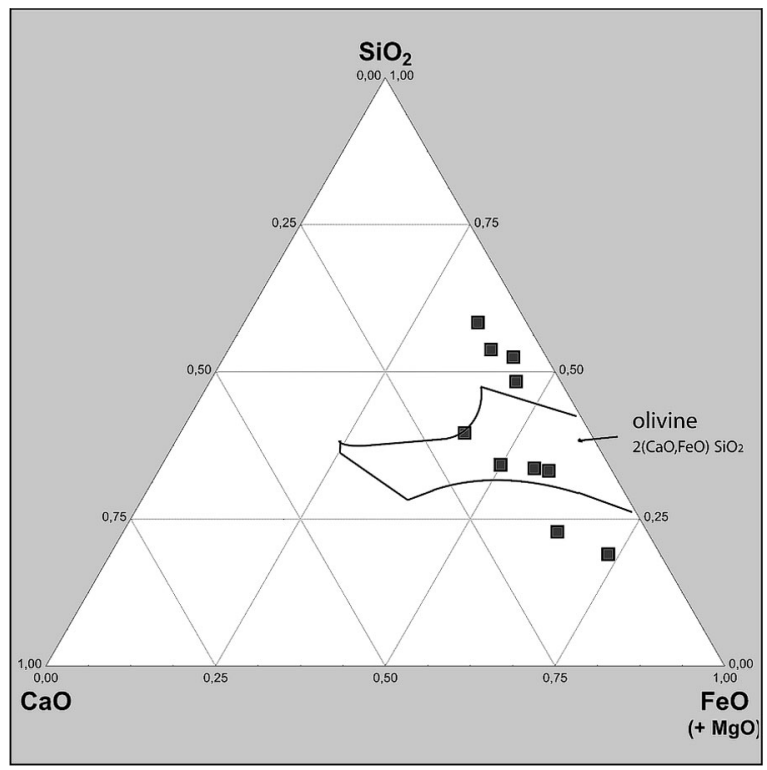

Fig. 8. Composition chimique élémentaire globale de 10 fragments de scories de La Cabane des Clausis, représentées dans le diagramme ternaire $\mathrm{FeO}(+\mathrm{MgO})-\mathrm{CaO}-\mathrm{SiO}_{2}$ (\% massiques, analyse par PIXE à partir de pastilles). La ligne en gras trace le liquidus de la fayalite, d'après (Muan and Osborn 1965).

zinc particulièrement élevée (3-6\% massique de $\mathrm{ZnO})$. De même, les microstructures montrent peu de variations (Fig. 9), avec un fort degré de cristallinité réparti en trois phases principales en sus des inclusions de cuivre métallique (pas ou très peu d'inclusions de sulfures de cuivre): des cristaux de fayalite riche en magnésium et en zinc (10-30\% et $1-2 \%$ massiques respectivement) aux textures morphologiques variables, des chaines massives de clino-pyroxenes riches en calcium $(10-20 \% \mathrm{Ca})$, et de fines dendrites à des polyèdres trapus de magnétite plus ou moins agrégés présents en des teneurs étonnamment peu variables (13 $\pm 2 \%$ en masse de magnétite). Les teneurs extrêmes en magnésium contenues dans la fayalite indiquent des températures minimales atteintes de l'ordre de $1.300^{\circ} \mathrm{C}$ (Deer et al. 1982). La viscosité des scories, estimée en utilisant le modèle de Lakatos (Lakatos et al. 1972), vaut $2,5 \pm 0,3$ Pa.s. On notera que la zone de morphologie prismatique si particulière au niveau macroscopique ne présente au niveau microstructural aucune spécificité par rapport au reste de la scorie. C'est du moins le cas pour les dix scories de la Cabane des Clausis étudiés par les auteurs. $\mathrm{Au}$ Vallon du Longet, on observe une microstruc-
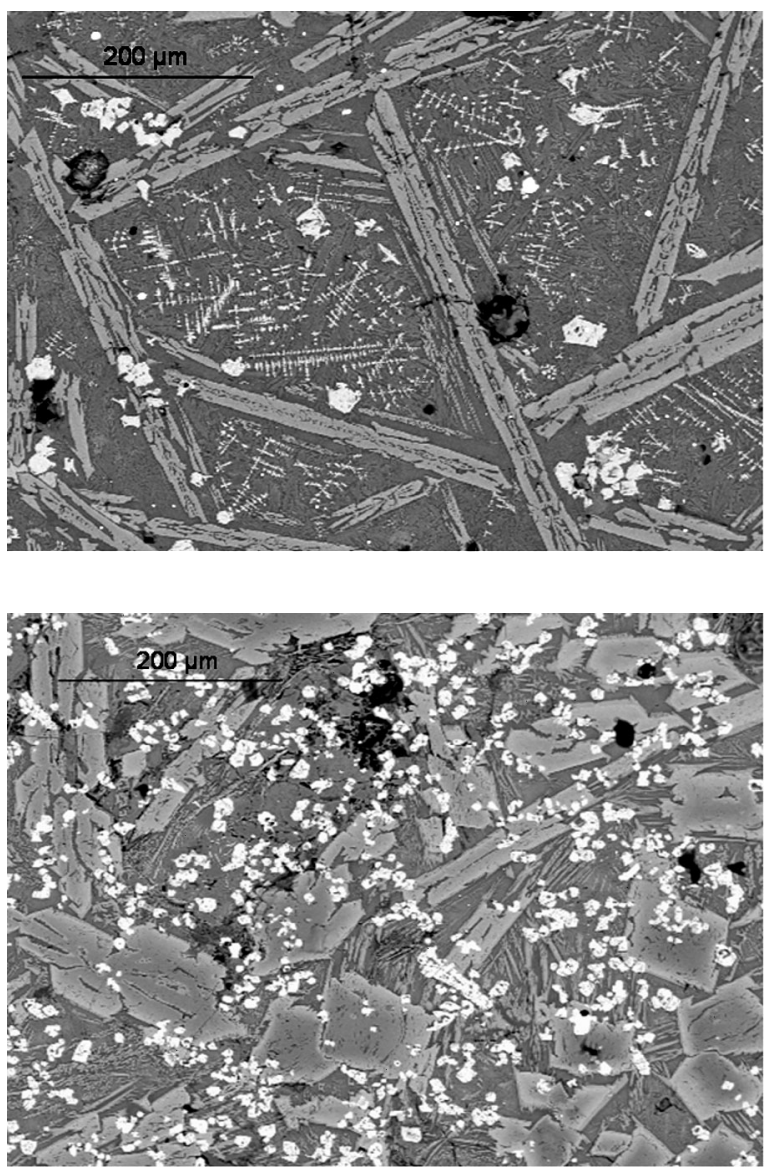

Fig. 9. Microstructures des scories de La Cabane des Clausis et du Vallon du Longet, montrant les 4 phases cristallines principales et les différentes textures morphologique prises par les olivines (fayalite): a) la fayalite cristallise sous forme de chaînes (larges aiguilles gris moyen) entremêlées de chaînes de clino-pyroxènes riches en calcium (gris foncé), la magnétite apparaît en fine dendrites et/ou en gros amas de polyèdres (girs clair), on remarque de petites inclusions de cuivre métallique (blanc), b) la fayalite cristallise ici en gros grains et montre un zonage de magnésium, les autres composés sont les mêmes que sur le cliché (a) (micrographies électroniques en électrons rétrodiffusés) sur coupes épaisses polies.

ture particulière dans les premiers millimètres à partir de la surface supérieure, marquée par des textures morphologiques de fayalite et de magnétite bien plus fines, voire la présence d'une couche de surface très riche en magnétite. Par ailleurs, il convient de signaler que les scories du Longet sont affectées par une altération en surface qui s'étend jusqu'à environ $1 \mathrm{~mm}$ dans la scorie, qui n'a épargné que les cristaux d'olivine. Un tel faciès n'a pas été observé aux Clausis. 


\section{RESTITUTION DES PROCEDES PYROMETALLURGIQUES}

\section{Un procédé "scorifiant"}

Les scories de Saint-Véran affichent une viscosité bien plus faible que celle des scories Chalcolithiques connues jusqu'alors (moins de $3 \mathrm{~Pa} . \mathrm{s}$ contre plus de $30 \mathrm{~Pa} . \mathrm{s}$ ), qui marquent un saut technologique très net par rapport à ce que l'on connaît des procédés en usage dans des contextes antérieurs de production à petite échelle (Bourgarit, 2007): on passe d'un procédé "non scorifiant" à un procédé où la synthèse des scories est bien maîtrisée. De fait, la question des paramètres responsables de la fluidité des scories est au cour de la caractérisation des procédés métallurgiques de Saint-Véran. Trois principaux paramètres peuvent être a priori incriminés: la composition chimique globale de la charge, les conditions d'oxydo-réduction, et la température qui prévalent dans le réacteur.

Les compositions élémentaires des dix scories de la Cabane des Clausis étudiées par les auteurs, comme celles du Vallon du Longet se situent toutes dans le domaine eutectique de la fayalite. Autrement dit, les températures de fusion de ces scories sont parmi les plus basses que l'on puisse espérer avec ce type de mélange. La composition particulière des scories a donc un rôle important sur les viscosités, à une température donnée (Bachmann et al. 1989). Ceci soulève la question de l'ajout délibéré de fondant pour optimiser la composition. De nombreux fragments de riébeckite $\left(\mathrm{Na}_{2} \mathrm{Fe}_{3}^{2+} \mathrm{Fe}_{2}^{3+}\left(\mathrm{Si}_{8} \mathrm{O}_{22}\right)(\mathrm{OH})_{2}\right)$ ont été retrouvés à $\mathrm{La}$ Cabane des Clausis. La distance importante des gisements de riébeckite, et la taille et de la quantité des fragments mis au jour sur le site métallurgique laissent penser que ce minéral a été amené délibérément, pour servir de fondant (Rostan et al. 1994a), sachant que, dans le cas présent, les propriétés de fondant de la riébeckite proviennent probablement plus du silicium (Si) que du sodium $(\mathrm{Na})$. La dispersion des compositions des scories (Fig. 8) pourrait à prime abord contredire l'aspect délibéré - et plus encore, contrôlé - de l'ajout de fondant. Cependant, il convient de noter que la composition de la riébeckite de Saint-Véran est elle-même très variable, depuis celle de la riébeckite pure à celle d'une riébeckite riche en quartzite et en oxydes de fer (principalement hématite, parfois magnétite): son ajout, même mesuré, peut très bien avoir induit la dispersion que l'on constate au sein des scories.

Pour que la composition chimique joue pleinement son rôle sur la viscosité de la scorie, encore faut il que les conditions d'oxydo-réduction soient adéquates. Notamment, le fer doit se trouver dans un état d'oxydation particulier (FeII) pour former la fayalite, faute de quoi de la magnétite risque d'apparaître, qui est connue pour augmenter notablement la viscosité des scories (Davenport et al. 2002). Dans les dix scories des Clausis étudiées par les auteurs, non seulement la quantité de magnétite apparaît comme étonnamment stable (13 \pm 2 $\mathrm{w} \%$ ), mais de plus cette quantité se situe juste en dessous du seuil critique défini par la métallurgie moderne, à savoir 16\% (Davenport et al. 2002) (p. 21), seuil qui constitue pour l'industrie moderne un enjeu majeur dans la définition des procédés. De quelle façon les anciens sont ils parvenus à contrôler cette quantité? Nous verrons dans ce qui suit que les conditions d'oxydo-réduction au sein de la structure pyrométallurgique ne sont pas les seuls paramètres responsables de la formation de la magnétite: le rapport des quantités de soufre et d'oxygène dans la charge initiale joue un rôle prépondérant. La composition de la charge pourrait donc de nouveau avoir une influence majeure.

D'après la composition en magnésium de la seule fayalite, dont la texture morphologique montre qu'elle a été formée au cours du refroidissement, une température d'au moins $1.300^{\circ} \mathrm{C}$ a été atteinte à Saint-Véran. Cependant, il est difficile de tenter une comparaison avec les températures des procédés Chalcolithiques, ces dernières étant très délicates à établir compte tenu de l'hétérogénéité des structures des scories associées. De nouvelles technologies ont-elles été mises en œuvre à Saint-Véran pour assurer sinon de plus hautes températures, du moins un accès plus aisé et mieux contrôlé à des températures avoisinant $1.300^{\circ} \mathrm{C}$ ? En l'absence de témoins archéologiques suffisants, l'influence de la morphologie des structures de combustion sur la température, via en particulier l'isolation thermique, ne peut malheureusement pas être discutée. On peut toutefois, à partir des reconstitutions des scories, estimer les diamètres internes des réacteurs comme étant supérieurs à $15-20 \mathrm{~cm}$. Or les diamètres internes des tuyères mises au jour à Saint-Véran sont en comparaison dérisoires (3-4 $\mathrm{mm})$, à tel point que la fonction de ces tuyères dans le procédé d'extraction peut être sujette à caution. Cepen- 
dant, l'efficacité de telles tuyères pour réduire du cuivre dans des structures aux diamètres internes analogues à ceux des fours de Saint-Véran a été démontrée archéologiquement et expérimentalement (Merkel et al. 1994), pour peu que plusieurs tuyères soient mises en œuvre simultanément. On remarquera que le système complet de ventilation associé à de telles tuyères, très fréquentes à l'âge du Bronze ancien et notamment dans les Alpes italiennes (Roden 1988), reste à ce jour à déterminer. Habituellement, ce type de petites tuyères est interprétés comme des embouts de tuyère à bouche (Rehder 1994), et c'est de cette façon que Merkel et al. (Merkel et al. 1994) ont proposé de reconstruire certains procédés précolombiens. Mais rien n'empêche a priori d'associer d'autres sources de ventilation, notamment des soufflets.

\section{Deux étapes pyrométallurgiques en continu dans un unique réacteur}

Les Plattenschlacke représentent quasiment le seul type de scories mises au jour à Saint-Véran, ce qui n'est pas le cas dans les régions alpines voisines d'Autriche et du Trentin italien, où pourtant la métallurgie de l'âge du Bronze est basée sur le même type de minerais, en l'occurrence des sulfures de cuivre mixtes: là, les scories du Chalcolithique et du début de l'âge du Bronze ancien sont principalement des scories très poreuses et volumineuses, en forme de "cake", les Schlackenkuchen (Anguilano et al. 2002; Artioli et al. 2007; Martinek and Sydow 2004; Mette 2003), tandis que les Plattenschlacke n'apparaissent qu'à partir de l'âge du Bronze moyen et sont systématiquement accompagnées de scories similaires à celles des périodes précédentes, à savoir les Schlackenkuchen, qui constituent sur certains sites le type majoritaire (Goldenberg 2004; Herdits 1993; Herdits 2003). A l'âge du Bronze final, du sable de scorie (Sandschlacke) vient compléter le mobilier détritique retrouvé sur les sites métallurgiques (Cierny et al. 2004, 2004b; Mette 2003). Signalons également la présence à la fin de l'âge du Bronze, mais cette fois de manière non systématique, de Laufschlacke, des scories ayant coulé hors de la structure de combustion (Doonan 1996). Le fait qu'un seul type de scorie ait été retrouvé à Saint Véran, et que la composition des scories étudiées varie relativement peu privilégient grandement l'hypothèse d'une unique étape pyrométallurgique. Certains témoignages éthnologiques (voir ci-après) montrent la faisabilité d'un tel procédé. De plus, quand bien même d'autres types de scories auraient été produits qui auraient échappé aux prospections et aux fouilles archéologiques - ce qui est très improbable, l'hypothèse d'un procédé en une étape pourrait rester valide, à l'image de la proposition de reconstitution du procédé à Acqua Fredda, Mitterberg, à l'âge du Bronze final, où trois différents types de scories ont été identifiés (Mette 2003).

Cette hypothèse est renforcée par le fait qu'aucune trace d'aire de grillage n'est attestée à La Cabane des Clausis, alors que ce type de structure est systématiquement retrouvé sur la plupart des sites plus tardifs d'Autriche et du Trentin cités plus haut. De fait, nous avons formulé et testé expérimentalement l'hypothèse (Bourgarit et al. 2002) d'un procédé où l'oxydation du minerai à l'état solide (grillage) et la fusion scorifiante à plus haute température prennent place dans un unique réacteur, et sont réalisées sans interruption l'une à la suite de l'autre. Là encore nous n'entrerons pas dans le détail des protocoles et résultats expérimentaux, pour simplement signaler les principaux résultats obtenus.

Dans un tel modèle, l'oxydation du sulfure de cuivre à l'air en haut du réacteur (le grillage) ne peut avoir qu'une durée très limitée $(30$ minutes tout au plus) avant que les grains de minerais tombent au fond du réacteur, dans une zone où l'air ne pénètre que difficilement et marquée par de plus hautes températures (autour de $1.200^{\circ} \mathrm{C}$, contre 600 à $800^{\circ} \mathrm{C}$ en haut). De fait, nous avons mesuré expérimentalement en laboratoire par analyse thermogravimétrique (Burger et al. 2010a) et sur le terrain, dans un vase-four et des conditions opératoires déduites des fouilles de l'habitat Néolithique de Al Claus (Carozza et al. 1997), les cinétiques d'oxydation de la chalcopyrite $\mathrm{CuFeS}_{2}$. Il en ressort qu'autant en four électrique de laboratoire, une oxydation complète peut être menée en environ $2 \mathrm{~h}$ à $700^{\circ} \mathrm{C}$, autant sur le terrain on parvient tout juste à éliminer $40 \%$ de soufre en quatre heures, c'est à dire à ne former que des oxydes de fer tout en conservant le cuivre intégralement sous forme de sulfure de type $\mathrm{Cu}_{2} \mathrm{~S}$. La raison principale (Burger et al. 2010b) en est que la cinétique d'oxydation est entièrement contrôlée par la diffusion de l'oxygène de l'air dans les couches de gaz liminaires, les- 
quelles sont très pauvres en oxygène en condition expérimentales de terrain (feu de charbon de bois). Or les simulations expérimentales de réduction à haute température menées sur des mélanges chalcopyrite/malachite (Bourgarit 2002; Burger et al. 2010b) ont montré que, dans des conditions modérément réductrices compatibles avec les conditions d'un réacteur du début de l'âge du Bronze $\left(10^{-8}<\mathrm{pO}_{2}<10^{-4}\right)$, il fallait dépasser une certaine quantité relative de malachite (en l'occurrence d'oxyde de cuivre) pour introduire suffisamment d'oxygène dans le système et parvenir à former une scorie comparable aux scories archéologiques, c'est-à-dire constituées de fayalite et de magnétite comme phases cristallines principales (Tab. 1). Dans la mesure où aucun oxyde de cuivre n'a pu être formé lors du grillage expérimental en extérieur, et donc que la quantité d'oxygène dans la charge initiale est insuffisante pour la réduction du cuivre, il s'avère qu'un tel procédé en une étape n'est pas possible pour réduire la chalcopyrite pure en cuivre.

Le procédé semble toutefois opérant si on procède en deux étapes: le produit du premier grillage, typiquement un sulfure de cuivre type
$\mathrm{Cu}_{2} \mathrm{~S}$, est récupéré avant l'étape haute température et grillé une seconde fois, pour subir dans la foulée une fusion scorifiante. C'est en tous cas ce que laissent entrevoir les résultats d'expérimentations préliminaires conduites par les auteurs sur le terrain, utilisant non plus de la chalcopyrite mais un minerai moins riche en soufre et fer, en l'occurrence de la bornite $\mathrm{Cu}_{5} \mathrm{FeS}_{4}$ : de relativement grandes quantités de métal ont été obtenues, avec qui plus est une seule étape de grillage de très faible durée (moins de 10 minutes). En tous cas, ces résultats montrent que dans le contexte particulier de Saint-Véran, où ce n'est pas de la chalcopyrite mais précisément de la bornite qui a été traitée, le procédé est techniquement faisable. A noter que d'après des études antérieures (Rosenqvist 2004), le sodium favorise grandement la séparation cuivre/fer à l'état solide lors des premières réactions de transformation du minerai et donc indirectement aussi la réduction du cuivre. Or la riébeckite $\left(\mathrm{Na}_{2} \mathrm{Fe}_{3}^{2+} \mathrm{Fe}_{2}^{3+}\left(\mathrm{Si}_{8} \mathrm{O}_{22}\right)(\mathrm{OH})_{2}\right)$ est a priori une source importante de sodium.

Un autre résultat intéressant des simulations expérimentales de l'étape subséquente de fusion scorifiante est d'avoir permis de mettre en place

\begin{tabular}{|c|c|c|c|c|c|c|c|}
\hline$-\log \left(\mathrm{pO}_{2}\right)$ & $\mathrm{O} / \mathrm{S}=\mathbf{0}$ & 0,8 & 2 & 2,5 & 3 & 4 & $\infty$ \\
\hline 1 (air) & $\begin{array}{l}\text { Delafossite } \\
\text { Magnétite } \\
\text { Cuprite }\end{array}$ & & $\begin{array}{c}\text { Delafossite } \\
\text { Cuprite } \\
\text { Ténorite }\end{array}$ & & & & \\
\hline 3 & $\begin{array}{l}\text { pas de } \\
\text { scorie } \\
\text { (matte) }\end{array}$ & $\begin{array}{l}\text { Quartz } \\
\text { fayalite }\end{array}$ & $\begin{array}{c}\text { Quartz } \\
\text { fayalite } \\
\text { magnétite }\end{array}$ & & $\begin{array}{l}\text { delafossite } \\
\text { magnétite }\end{array}$ & & cuprite \\
\hline 4 & & & $\begin{array}{c}\text { Quartz } \\
\text { fayalite } \\
\text { magnétite }\end{array}$ & $\begin{array}{l}\text { delafossite } \\
\text { magnétite }\end{array}$ & $\begin{array}{l}\text { delafossite } \\
\text { magnétite }\end{array}$ & & \\
\hline 7 & & & $\begin{array}{l}\text { Quartz } \\
\text { fayalite } \\
\text { magnétite }\end{array}$ & $\begin{array}{l}\text { Quartz } \\
\text { fayalite } \\
\text { magnétite }\end{array}$ & $\begin{array}{l}\text { delafossite } \\
\text { magnétite }\end{array}$ & & \\
\hline 10 & & & & $\begin{array}{l}\text { Quartz } \\
\text { fayalite } \\
\text { magnétite }\end{array}$ & & & \\
\hline
\end{tabular}

Tab. 1. Nature des produits synthétisés lors des simulations expérimentales de réduction de minerais de cuivre menées en four électrique de laboratoire, en isothermes de 30 minutes à $1.200^{\circ} \mathrm{C}$. Les deux paramètres testés sont le rapport molaire malachite/chalcopyrite dans la charge initiale (exprimé en rapport oxygène/soufre), et la pression partielle d'oxygène dans le réacteur imposée par le balayage de gaz tampon aux proportions variables de $\mathrm{CO}$ et $\mathrm{CO}_{2}$. Les résultats présentés mentionnent la présence ou non des trois phases cuivre métallique/matte (sulfure de cuivre et fer)/scorie. La nature des phases cristallisées dans la scorie est également précisée. D’après (Burger 2008; Burger et al. 2010b). 
une méthodologie capable de distinguer, à partir de la caractérisation analytique des scories archéologiques, la part des deux sources possibles d'oxygène (Burger et al. 2010b), en l'occurrence l'oxygène apporté par l'air et celui apporté par les oxydes, notamment de cuivre, issus soit du minerais soit de l'étape de grillage. En effet, jusqu'à présent n'était considéré que l'oxygène gazeux comme responsable des conditions d'oxydo réduction, sans prendre en compte le rôle non négligeable des quantités relatives d'oxygène et de soufre apportées par le minerai traité. Or nous avons montré (Burger et al. 2010b) combien cette négligence peut, dans certains cas, mener à des estimations totalement erronées. Ainsi, les scories du site Enéolithique de Riparo Gaban dans le Trentin italien ne contiennent du fer qu'essentiellement sous forme de fayalite: la seule utilisation du diagramme de phase à l'équilibre $\mathrm{FeO}$ $\mathrm{Fe}_{2} \mathrm{O}_{3}-\mathrm{SiO}_{2}$ (Muan 1955) indiquerait, pour une température de $1.200^{\circ} \mathrm{C}$, une $\mathrm{pO}_{2}$ de l'ordre de $10^{-10} \mathrm{~atm}$. Or le très bas rapport $\mathrm{O} / \mathrm{S}(\sim 0.6)$ estimé à partir des quantités de soufre restantes conduit en réalité à une une $\mathrm{pO}_{2}$ de l'ordre de $10^{-4} \mathrm{~atm}$.

\section{ORGANISATION DE LA PRODUCTION}

\section{Une multitude de sites métallurgiques fonctionnant sur de courtes durées}

A partir de l'étude dimensionnelle des mines anciennes et des scories, les taux de production à Saint-Véran à l'âge du Bronze ancien ont pu être estimés (Bourgarit et al. 2008; Rostan et al. 2002). Nous présentons ci-dessous un résumé des différents calculs et estimations réalisées.

Avec un diamètre estimé de $150 \mathrm{~mm}$, une épaisseur de $15 \mathrm{~mm}$, et une densité de $3,5 \mathrm{~g} . \mathrm{cm}^{-3}$, une scorie de Saint-Véran pèse autour de $930 \mathrm{~g}$ : c'est la quantité de déchet générée par une opération de réduction. En considérant que le fer contenu dans la scorie ne provient que de la bornite, le calcul précédent montre qu'environ $2,5 \mathrm{~kg}$ de bornite étaient traités au cours d'une opération. Il a été montré que la morphologie prismatique particulière des sections des scories indiquait que la cadence et donc le taux de production étaient un enjeu majeur à Saint-Véran à l'âge du Bronze ancien. Il a été également estimé que l'opération dans sa totalité pouvait durer moins de deux heures. En considérant donc une durée moyenne d'opération de deux heures, on peut raisonnablement estimer que quatre opérations de réduction pouvaient être réalisées en une journée, d'autant que la phase minéralurgique de tri de minerai devait être très facile et rapide compte tenu de la richesse du minerai exploité. Lors de nos expérimentations utilisant le minerai de Saint-Véran, une charge de $500 \mathrm{~g}$ de bornite pouvait être préparée en moins de 30 minutes. Par conséquent $10 \mathrm{~kg}$ de bornite pouvaient être réduits au cours d'une journée par un atelier métallurgique.

Côté mines, il a été montré que le taux d'extraction minière était estimé à $4 \mathrm{~m}^{3}$ par mois, soit avec un rapport massique bornite/roche encaissant de 1/12 un taux de production de bornite de $25 \mathrm{~kg}$ par jour. Par conséquent, un atelier comme celui de La Cabane des Clausis était plus ou moins en mesure d'absorber la production minière journalière. Bien que les activités minières et métallurgiques n'aient probablement pas été en phase à l'échelle d'une année en raison d'interruptions saisonnières décalées - l'hiver pour l'atelier et le printemps voire l'été pour la mine, le calcul réalisé ci-dessus tend à montrer qu'un seul atelier métallurgique pouvait suffire à assurer la production de cuivre de tout le gisement.

S'agissant de la production totale du site de Saint-Véran à l'âge du Bronze ancien, un total d'environ $10.000 \mathrm{~m}^{3}$ de minerais avec sa gangue ont été extraits de la mine durant toute la durée de son exploitation, qui correspondent à quelques $2.000 \mathrm{t}$ de bornite. Or quelques $100 \mathrm{~kg}$ de scories ont été retrouvés à la Cabane des Clausis, le plus gros gisement de scories trouvé à ce jour. Ceci correspond, on vient de le voir, à $250 \mathrm{~kg}$ de bornite traités sur toute la durée d'occupation du site, soit une quantité dérisoire en regard des 2.000 tonnes de bornites extraites des mines. De plus, cela rend compte d'une durée d'occupation très courte: à un rythme de $10 \mathrm{~kg}$ de bornite réduits au cours d'une journée, la Cabane des Clausis serait restée en activité pendant 25 jours, alors que les mines auraient été exploités pendant 200 ans (400 ans si on considère une activité saisonnière de 6 mois par an)! Une telle différence ne peut se justifier que si de nombreux autres sites de métallurgie ont été associés à l'exploitation minière, sachant que des sites de capacité véritablement supérieure à celle de la Cabane des Clausis sont à ce jour fortement improbables au vu des nombreuses prospections réalisées dans tout le secteur. De fait, il est fort probable que tous les ate- 
liers métallurgiques aient eu des activités très localisées dans le temps, de l'ordre de la saison, phénomène tout à fait particulier qu'il conviendra d'étudier. A noter que les faciès de fracture si particuliers des fragments de scories des Clausis n'ont quasiment pas été observés au Vallon du Longet, bien qu'on ne puisse pas exclure que la corrosion ait érodé ces reliefs. Ceci, combiné aux microstructures spécifiques observées en surface des seules scories du Longet pourrait signer des conditions de refroidissement différentes et donc des modes opératoires légèrement modifiés. De plus, il est possible que les structures de combustion aient différé d'un site à l'autre, eu égard aux différences de morphologie pressenties. L'origine de ces légères variations, si elles se confirmaient, resterait à déterminer. Plusieurs hypothèses seraient à tester, à commencer par celle d'un décalage chronologique.

Par ailleurs, les choix des emplacements sont aussi à éclaircir, à l'image du site du Vallon du Longet pour lequel le minerai devait franchir un col avant d'être traité.

\section{Diffusion de la production}

Les calculs précédents (Rostan et al. 2002) estiment une production de l'ordre de $1.400 \mathrm{t}$ de cuivre métallique pendant plusieurs centaines d'années. Or la destination de ce métal reste aujourd'hui mal connue. Les mêmes auteurs ont souligné l'importance culturelle exercée par le complexe italique, à travers en particulier la présence de pétroglyphes à proximité de Saint-Véran, représentant un personnage et deux poignards de type Remedello (à lame triangulaire à base droite et pommeau). De même, l'abri des Oullas, situé dans la haute vallée de l'Ubaye (Alpes-de-Haute-Provence) à environ $6 \mathrm{~km}$ de la zone métallurgique de Saint-Véran, témoigne largement de cette influence. Il suffit, de plus, de rappeler que le site minier de Saint-Véran n'est distant que de $3 \mathrm{~km}$ de l'actuelle frontière franco-italienne (Fig. 1). En particulier, le col de Longet constitue, à une altitude de $2.650 \mathrm{~m}$, une porte ouverte sur le Val Varaita (Cuneo). L'hypothèse est donc plus que tentante, et n'attend plus que d'être testée par l'analyse chimique, à l'image du travail conduit récemment sur le mobilier métallique du dépôt tout proche de Monte Cavanero (Artioli et al. 2009), daté de l'âge du Bronze fi- nal. Dans ce dernier cas, les analyses ont permis de clairement identifier les gisements piémontais comme source principale de cuivre.

\section{CONCLUSION}

Le résultat le plus important des vingt années de recherche pluridisciplinaire menée autour du gisement de Saint-Véran est sans doute la mise en évidence du caractère exceptionnel du site, témoin de la mise en place d'une production de cuivre à grande échelle dès la fin du troisième millénaire avant notre ère. De plus, cas peu fréquent pour les périodes protohistoriques, non seulement le volume de cette production a pu être estimé, mais les calculs de capacité de production ont permis d'associer étroitement activités minières et métallurgiques. Avec cette apparition précoce d'un système de production de masse coïncide la mise en œuvre de technologies minières et métallurgiques novatrices, qui marquent un tournant dans l'histoire de la métallurgie du cuivre. C'est notamment la maîtrise nouvelle d'une métallurgie "scorifiante", avec l'attestation la plus ancienne des scories de type Plattenschlacke dans l'histoire de la métallurgie du cuivre.

Ceci étant, les données archéologiques actuelles ont révélé un décalage marqué entre les quantités importantes de minerais extraites et les quantités dérisoires de cuivre retrouvées, directement ou indirectement. En d'autre terme, on ne connaît pas aujourd'hui la destination de la bornite de Saint-Véran: ni la destination première, à savoir les lieux de sa transformation en métal, ni la destination finale, en l'occurrence l'aire de diffusion du métal produit. D'une part, les deux seuls sites métallurgiques fouillés associés aux mines témoignent d'une production faible, et donc en particulier très limitée dans le temps: les estimations montrent que l'activité d'un site n'a pas duré plus d'une saison, alors que l'exploitation minière s'est développée sur plusieurs centaines d'années. Reste donc à localiser au moins part des centaines de sites équivalents, probablement répartis autour des mines à moyenne distance (l'aire de traitement du Vallon du Longet se situe à environ une demi-heure de marche de la mine de la Tranchée des Anciens), voire dans un périmètre bien plus large puisque, pour l'heure, aucun élément ne permet d'écarter l'hypothèse d'une exportation de minerai à longue distance. 
Et surtout, reste à comprendre le pourquoi d'un tel éclatement de l'activité de traitement métallurgique autour d'une source minière elle étonnamment circonscrite dans l'espace. D'autre part, très peu de mobilier métallique est aujourd'hui à mettre en face des quantités importantes de cuivre produites. En particulier, la nature et les dimensions de l'aire de diffusion du métal de Saint-Véran sont encore à déterminer, bien que de nombreux indices tendent à privilégier l'hypothèse d'une destination principalement italique.

Le caractère exceptionnel du site a naturellement conduit les auteurs à s'interroger sur les facteurs potentiellement favorables à la mise en place d'une production de cuivre de masse au début de l'âge du Bronze ancien. Plusieurs facteurs naturels ont pu être identifiés à Saint-Véran (Bourgarit et al. 2008), dont la combinaison est unique pour les Alpes, à cette époque. Ainsi, si la présence de cuivre natif a pu initier les premières exploitations intensives, le gisement de bornite particulièrement massif a constitué une réserve importante de minerai facile à extraire et à trier. De plus, la richesse en cuivre de la bornite où fer et soufre sont en des teneurs bien moindres que dans la chalcopyrite, a certainement facilité la transition technologique et permis d'augmenter fortement les rendements de transformation. Pour autant, la question des dynamiques d'implantation d'une telle activité au début de l'âge du Bronze ancien dans des zones de haute montagne demeure entière, à l'heure où les exemples se multiplient dans les alpes occidentales. Plus au nord en effet, dans le massif des Grandes Rousses, la découverte récente d'un vaste secteur minier entre 2250 et $2600 \mathrm{~m}$ d'altitude (Bailly-Maitre and Gonon 2008) et l'identification de pollutions liées aux activités métallurgiques (cuivre et plomb) en milieu lacustre proglaciaire d'altitude (Lac Bramant, $2.500 \mathrm{~m}$ ) (Guyard et al. 2007), témoignent d'une exploitation massive des minerais de cuivre durant le début de l'âge du Bronze (2200-1650 BC). Or, si on connait encore mal la nature et la structure des minéralisations aux Rousses, il est certain que les deux éléments favorables de Saint-Véran - un minerai hypo sulfuré et pauvre en fer se développant selon un caractère exceptionnellement massif - n'y sont pas. Le rôle de la nature particulière du gisement métallifère de Saint-Véran doit donc être nuancé pour expliquer l'implantation et le développement de l'activité minière et métallurgique. Déjà, des voies alternatives sont proposées (Carozza et al. 2010a, Carozza et al. 2009) suite à l'observation pour ces périodes d'épisodes de déprise glaciaire susceptibles d'avoir pu offrir des accès jusqu'alors inédits aux ressources métallifères d'altitude. Il conviendra bien entendu de confronter à ces seuls déterminismes naturels d'éventuelles influences sociales, politiques voire culturelles.

\section{BIBLIOGRAPHY}

Ancel, B. 1997: "La mine de cuivre des Clausis à Saint-Véran: apport des source écrites et relevé des travaux préhistoriques souterrains". Archéologie en Languedoc 21: 121-128.

Anguilano, L.; Angelini, I.; Artioli, G.; Moroni, M.; Baumgarten, B. and Oberrauch, H. 2002: "Smelting Slags from Copper and Bronze Age archaeological sites in Trentino and Alto Adige". En C. D'Amico (ed.): Atti del II Congresso Nazionale di Archeometria (Bologna 2002). Patron. Bologna: 627-638.

Artioli, G.; Angelini, I.; Burger, E. and Bourgarit, D. 2009: "Petrographic and Chemical Investigations of the Earliest Copper Smelting Slags in Italy: towards a Reconstruction of the Beginning of Copper Metallurgy". In Proceedings of the 2nd International Conference Archaeometallurgy in Europe (Aquileia 2007), AIM Associazione Italiana di Metallurgia. Milano: 12-20.

Artioli, G.; Angelini, I.; Giunti, I.; Omenetto, P. and Villa, I.M. 2009: "La provenienza del metallo degli oggetti di Monte Cavanero: considerazioni basate sugli isoptopi del $\mathrm{Pb}$ esulla geochimica delle mineralizzazioni cuprifere limitrofe". En M. Venturino Gambari (ed.): Il ripostiglio del Monte Cavanero di Chiusa di Pesio. Soprintendenza per i Beni Archeologici del Piemonte. Alessandria: 167-178.

Bachmann, H.G.; Lutz, C. and Thiemann, U. 1989: "Schlakenviskositäten: experimentelle Bestimmung und theoritische Berechnung". En A. Hauptmannn, E. Pernicka and G.A. Wagner (eds.): Proc. Int. Symp. Old World Archaeometallurgy (Heidelberg 1987). Deutches Bergbau-Museum. Bochum: 137-140.

Bailly-Maitre, M. C. and Gonon, T. 2008: "L'exploitation de la chalcopyrite à l'âge du Bronze dans le massif des Rousses en Oisans (Isère): premiers éléments". En H. Richard and D. García (dir.): Le peuplement de l'arc alpin. CTHS. Paris: 207-223.

Barge, H. 1997: "L'installation métallurgique préhistorique de la cabane des Clausis à Saint Véran (Hautes Alpes)". Archéologie en Languedoc 21: 99-110. 
Bourgarit, D. and Mille, B. 1997: "La métallurgie chalcolithique de Cabrières: confrontation des données expérimentales et archéologiques en laboratoire". Archéologie en Languedoc 21: 51-63.

Bourgarit, D. and Mille, B. 2005: "Nouvelles données sur l'atelier métallurgique de la Capitelle du Broum (district minier de Cabrières, Hérault, France): la transformation de minerais de cuivre à base de sulfures se précise!". En Ambert, P. and Vacquer, J. (ed.): Mémoire XXXVII de la Société Préhistorique Française, La première métallurgie en France et dans les pays limitrophes. Société Préhistorique Française. Carcassonne: 97-108.

Bourgarit, D.; Mille, B.; Burens, A. y Carozza, L. 22-26 april 2002: "Smelting of chalcopyrite during chalcolithic times: some have done it in ceramic pots as vase-furnaces". En H. Kars and E. Burke (eds.): 33rd International Symposium on Archaeometry (Amsterdam 2002). Vrije Universiteit. Amsterdam: 297-302.

Bourgarit, D.; Mille, B.; Prange, M.; Ambert, P. and Hauptmann, A. 24-26 sept 2003: "Chalcolithic Fahlore Smelting at Cabrières: Reconstruction of Smelting Processes by Archaeometallurgical Finds". Archaeometallurgy in Europe (Milano 2003). En (ed.) Associazione Italiana di Metallurgia. City: 431-440.

Bourgarit, D.; Rostan, P.; Burger, E.; Carozza, L. and Artioli, G. 2008: "The beginning of copper mass production in the western Alps: the Saint Véran mining area reconsidered". Historical Metallurgy 42(1): 1-11.

Burger, E.; Bourgarit, D.; Frotté, V. and Pilon, F. 2010a: "Kinetics of iron-copper sulphides oxidation in relation to protohistoric copper smelting". Journal of thermal analysis. DOI 10.1007/510973010-0926.

Burger, E.; Bourgarit, D.; Wattiaux, A. and Fialin, M. 2010b: "The reconstruction of the first copper smelting processes in Europe during the 4th to the 3rd millennium BC: Where does the oxygen come from?". Applied Physics A. 100 (3): 713-724.

Carozza, L.; Bourgarit, D. and Mille, B. 1997: 'L'habitat et l'atelier de métallurgiste chalcolithique d'Al Claus: analyse et interprétation des témoins d'activité métallurgique". Archéologie en Languedoc 21: 147-160.

Carozza, L.; Chapron, E.; Simmoneau, A.; Mille, B.; Guyard, H.; St Onge, G.; Rostan, P.; Bourgarit, D. and Burens, A. 2010b: "Glacial fluctuations and exploitation of copper resources in high mountain during the late Neolithic and Bronze Age in the French Alps (2500-1500 bc)". En Special Conference of the SFB HiMAT on Mining in European History (Innsbrück, Austria 2009). 81-90.

Carozza, L. and Mille, B. 2007: "Chalcolithique et complexification sociale: quelle place pour le métal dans la définition du processus de mutation des sociétés de la fin du Néolithique en France?". En J. d. Guilaine (ed.): Le Chalcolithique et la construction des inégalités, tome 1, le continent européen, séminaires du collège de France. Errance. Paris: 195-232.

Carozza, L.; Mille, B.; Rostan, P.; Bourgarit, D. and Burens, A. sous presse: "Mine et métallurgie en haute montagne durant la fin du Néolithique et le début de l'âge du Bronze: l'exemple de Saint-Véran en Haut-Queyras (Hautes-Alpes, France)". En actes du colloque L'Eta del Rame in Italia (Bologne 2009). Instituto Italiano de Prehistoria et Protohistoria (IIPP). Bologna.

Carozza, L.; Rostan, P.; Bourgarit, D.; Mille, B.; Coquinot, Y.; Burens, A. and Escanilla Artigas, N. sous presse: "Un site métallurgique du Bronze ancien dans le vallon du Longet à Molines-en-Queyras (Hautes-Alpes): caractérisation du contexte archéologique et des déchets liés aux activités de métallurgie extractive". En int. Conf. Archéologie de l'espace montagnard: confrontation d'expériences européennes (Gap 2008). Musée-Muséum Départemental. Gap. Errance. Paris: 261-281.

Cierny, J.; Marzatico, F.; Perini, R. and Weisgerber, G. 2004: "La riduzione del rame in località Acqua Fredda al Passo del Redebus (Trentino) nell'età del Bronzo Recente e Finale". En G. Weisgerber and G. Goldenberg (ed.): Alpenkupfer - Rame delle Alpi. Deutsches Bergbau Museum. Bochum: 125-154.

Cierny, J.; Marzatico, F.; Perini, R. and Weisgerber, G. 2004b: "Der spätbronzezeitliche Verhüttungsplatz Acqua Fredda am Passo Redebus (Trentino)". En G. Weisgerber and G. Goldenberg (eds.): Alpenkupfer - Rame delle Alpi. Deutsches Bergbau Museum. Bochum: 155-164.

Davenport, W.G.; King, M.; Schlesinger, M. and Biswas, A.K. 2002: Extractive Metallurgy of copper. Elsevier. Oxford.

Deer, W.A.; Howie, R.A. and Zussman, J. 1982: Rock-forming minerals: Vol. 1A. second edition: orthosilicates. Longman Scientific And Technical. Harlow.

Gattiglia, A. and Rossi, M. 1995: "Les céramiques de la mine préhistorique de Saint-Véran (Hautes-Alpes)". Bulletin de la Société Préhistorique Française, 92(4): 509-518.

Goldenberg, G. 2004: "Ein Verhüttungsplatz der mittleren Bronzezeit bei Jochberg (Nordtirol)". En G. Weisgerber and G. Goldenberg (eds.): Alpenkupfer - Rame delle Alpi. Deutsches Bergbau Museum. Bochum: 165-176.

Guyard, H.; Chapron, E.; St-Onge, G.; Anselmetti, F.S.; Arnaud, F.; Magand, O.; Francus, P. and Mélières, M.A. 2007: "High-altitude varve records of abrupt environmental changes and mining activity over the last 4000 years in the Western French Alps

T. P., 67, N. ${ }^{\circ}$ 2, julio-diciembre 2010, pp. 269-285, ISSN: 0082-5638

doi: $10.3989 /$ tp.2010.10039 
(Lake Bramant, Grandes Rousses Massif)". Quaternary Science Reviews 26: 2644-2660.

Hauptmann, A. 2000: Zur frühen Metallurgie des Kupfers in Fenan/Jordanien. Deutsches Bergbau Museum. Bochum.

Herdits 1993: "Zum Beginn experimentalarchäologischer Untersuchungen einer bronzezeitlichen Kupferverhüttungsanlage in Mühlbach, Salzburg". Archaeologia Austriaca, 77: 31-38.

Herdits, H. 2003: "Bronze Age smelting site in the Mitterberg mining area in Austria". En P. Craddock and J. Lang (eds.): Mining and metal production through the ages. British Museum Press. London: 69-75.

Lakatos, T.; Johansson, L.-G. and B., S. 1972: "Viscosity temperature relations in the glass system $\mathrm{SiO}_{2}-\mathrm{Al}_{2} \mathrm{O}_{3}-\mathrm{Na}_{2} \mathrm{O}-\mathrm{K}_{2} \mathrm{O}-\mathrm{CaO}-\mathrm{MgO}$ in the composition range of technical glasses". Glass Technology 13(3): 88-95.

Martinek, K.P. and Sydow, W. 2004: "Frühbronzezeitliche Kupfermetallurgie im Unterinntal (Nordtirol)". En G. Weisgerber and G. Goldenberg (eds.): Alpenkupfer - Rame delle Alpi. Deutsches Bergbau Museum. Bochum: 199-211.

Merkel, J.F.; Shimada, I.; Swann, C.P. and Doonann, R. 1994: "Prehispanic copper alloy production at Batan Grande, Peru: interpretation of the analytical data for ore sample". En D. Scott and P. Meyers (eds.): Archaeometry of pre-columbian sites and artefacts. Getty Conservation Institute. Los Angeles: 199-227.

Metten, B. 2003: "Beitrag zur spätbronzezeitlichen Kupfermetallurgie im Trentino (Südalpen) im Vergleich mit anderen prähistorischen Kupferschlacken aus dem Alpenraum". Metalla 10 1/2: 1-122.

Mille, B. and Carozza, L. 2009: "Moving into the Metal Ages: The Social Importance of Metal at the End of the Neolithic period in France". En T.L. Kienlin and B.W. Roberts (eds.): Metals and Society, studies in honour of Barbara S. Ottaway. Habelt. Bonn: 143-171.

Muan, A. 1955: "Phase equilibria in the system $\mathrm{FeO}-\mathrm{Fe}_{2} \mathrm{O}_{3}-\mathrm{SiO}_{2}$ ". Trans. (TMS) AIME, 203: 965-976.
Muan, A. and Osborn, E.F. 1965: Phase equilibria among oxides in steelmaking. Addison-Wesley. Reading, Massachussetts.

Ploquin, A.; Happ, J.; Barge, H. and Bourhis, J.-R. 1997: "Scories archéologiques et reconstitution expérimentale de réduction de sulfure de cuivre (minerai de St.-Véran, Hautes-Alpes): prémices d'une approche pétrographique". Archéologie en Languedoc 21: 111-120.

Rehder, J.E. 1994: "Blowpipes Versus Bellows in Ancient Metallurgy". Journal of Field Archaeology 21(3): 345-350.

Roden, C. 1988: "Blasrohrdüsen. Ein archäologischer Exkurs zur Pyrotechnologie des Chalkolithikmus und der Bronzezeit". Der Anschnitt 40(3): 62-82.

Rosenqvist, T. 2004: Principles of extractive metallurgy (Second edition). Tapir Academic Press. Trondheim.

Rostan, P.; Gattiglia, A. and Rossi, M. 1994a: "Ricerche sulle miniere e sulla metallurgia dell'eta del Bronzo nel Briançonnais (Hautes-Alpes, Francia)". En F. Zampicinini (ed.): De Re Metallica, miniere e materie prime alle soglie del $3{ }^{\circ}$ millennio. 173-181.

Rostan, P. and Malaterre, J. 1994b: Les Clausis-Pinilière: ensemble minier, Saint-Véran. Bilan Scientifique. Marseille.

Rostan, P. and Mari, G. 2005: "L'exploitation protohistorique de cuivre natif de Roua (Daluis et Guillaumes, Alpes-Maritimes)". En P. Ambert and J. Vacquer (dirs.): Actes de la conférence. La première métallurgie en France et dans les pays limitrophes (Carcassonne 2002). Société Préhistorique Française. Paris: 139-149.

Rostan, P.; Rossi, M. and Gattiglia, A.: "Approche économique et industrielle du complexe minier et métallurgique de Saint-Véran (Hautes-Alpes) dans le contexte de l'Age du Bronze des Alpes du Sud". En Actes de la conférence Les Alpes dans L'Antiquité (Tende 2002) Société Valdôtaine de Préhistoire et d'Archéologie. Aoste: 77-96.

Rothenberg, B. 1990: The Ancient Metallurgy of Copper. Institute of Archaeology. London. 